\title{
La peligrosidad sísmica y el factor de riesgo
}

\section{Seismic hazard and risk factor}

\author{
B. Perepérez ${ }^{(*)}$
}

\section{RESUMEN}

Los terremotos se encuentran entre los desastres naturales que causan mayor número de víctimas y pérdidas económicas más cuantiosas. Este trabajo repasa el concepto de riesgo sísmico, la peligrosidad sísmica de la Península Ibérica y aspectos básicos de las normas sísmicas española (NCSE-02) y europea (EN 1998-1). Se han deducido relaciones analíticas entre el factor de importancia del Eurocódigo 8 (factor de riesgo en NCSE-02) y el período medio de retorno, el período de exposición y la probabilidad de superación durante éste del parámetro elegido de la intensidad sísmica. Se han efectuado consideraciones acerca del requisito de limitación de los daños y de la importancia de abordar su comprobación con más rigor.

Palabras clave: Peligrosidad sísmica; análisis sísmico; factor de riesgo; factor de importancia; período medio de retorno; período de exposición; probabilidad de superación.

\section{ABSTRACT}

Earthquakes are among the natural hazards which cause the greater number of human victims as well as the greater economic losses. This paper reviews the seismic risk concept, the Spanish seismic hazard and the basic aspects of the Spanish (NCSE-O2) and European (EN1998-1) standards. Some analytic relationships have been deduced between the importance factor of the Eurocode 8 (risk factor in the NCSE-O2) and the mean return period, as well as between the exposure time and the probability of exceedance during this time of the chosen seismic intensity parameter. Some considerations have been made about the damage limitation requirement and the importance of addressing to it a more rigorously checking.

Keywords: Seismic hazard; seismic design; risk factor; importance factor; return period; exposure time; probability of exceedance.

(*) ETSA - Universitat Politècnica de València (España).

Persona de contacto/Corresponding Author: bpereper@csa.upv.es (B. Perepérez)

Cómo citar este artículo/Citation: Perepérez, B. (2014). La peligrosidad sísmica y el factor de riesgo. Informes de la Construcción, 66(534): e018, doi: http://dx.doi.org/10.3989/ic.13.018.

Licencia/License: Salvo indicación contraria, todos los contenidos de la edición electrónica de Informes de la Construcción se distribuyen bajo una licencia de uso y distribución Creative Commons Reconocimiento no Comercial 3.o. España (cc-by-nc). 


\section{INTRODUCCIÓN}

Hay diversos fenómenos naturales que son capaces de representar una amenaza seria para los seres humanos y sus propiedades. De entre ellos, los que causan un mayor número de víctimas y de pérdidas materiales por unidad de tiempo son, probablemente, los terremotos (1).

Según la Estrategia Internacional para la Reducción de Desastres de la ONU (2), los fenómenos más mortíferos en los últimos veinte años han sido los terremotos, con casi 760,000 víctimas. En cuanto a las pérdidas económicas, las mayores de este mismo período las causaron las inundaciones y los sismos, ascendiendo, respectivamente, a 720,000 y 636,000 millones de dólares USA. No obstante, incluso el gran público tiene la percepción acertada de que seísmos de características similares causan pérdidas de magnitud muy distinta en función del lugar en el cual acontecen. Este hecho pone de manifiesto la necesidad de cuantificar el riesgo sísmico de cada lugar, concepto nada simple y que, con frecuencia, se utiliza con un rigor insuficiente (3).

El documento Natural Disasters and Vulnerability Analysis (4) divide los fenómenos naturales capaces de provocar desastres en tres categorías, a saber: fenómenos meteorológicos e hidrológicos, terremotos y volcanes. Propone que se apliquen a todos los fenómenos mencionados las definiciones siguientes:

- Peligrosidad o amenaza «H» (natural hazard): Probabilidad de que ocurra un fenómeno natural potencialmente desastroso en un lugar determinado y dentro de un período de exposición definido previamente.

- Vulnerabilidad «V» (vulnerability): Nivel de la pérdida ocasionada en un elemento o conjunto de elementos amenazados si ocurre un fenómeno natural de una magnitud determinada. Se valora en una escala que va del o al 1: sin daños y pérdida total, respectivamente.

- Elementos amenazados «E» (elements at risk): Valor económico de la población, de los edificios y obras civiles, de las actividades económicas, de los servicios públicos e infraestructuras, etc., amenazados dentro de un área geográfica determinada, incluidas las pérdidas económicas indirectas presentes y futuras.

- Riesgo «R» (risk): número de muertos y de heridos, pérdidas materiales e interrupción de la actividad económica a causa de cierto fenómeno natural. Por consiguiente, el riesgo es función de la peligrosidad, de la vulnerabilidad y de los elementos amenazados [1].

$$
R=R(H, V, E)
$$

\section{LA PELIGROSIDAD SÍSMICA EN ESPAÑA}

La peligrosidad sísmica se ha definido como la probabilidad de que se supere un determinado valor de la variable sísmica «IM» en un período de «T» años (4). Por lo tanto, la peligrosidad o amenaza sísmica es un concepto probabilista y sismológico (5), que no hay que confundir con los conceptos de vulnerabilidad o de riesgo sísmicos (ver apartado 1).

La peligrosidad sísmica de la península Ibérica no es despreciable, pues se halla ubicada en el borde sudoeste de la placa Euroasiática en su colisión con la placa Africana (6) (1). Los terremotos más destructivos de la península Ibérica ocurrieron antes del siglo XX. Desde el punto de vista de la peligrosidad sísmica, revisten gran importancia los acaecidos en el denominado período histórico, que se extiende, aproximadamente, entre los años 1000 y 1900 (7), aunque se puede extender hasta 1921 (8). La Tabla 1 está basada en el trabajo de Martínez Solares y Mezcua (9) y en los datos de 2012 del Servicio de Información Sísmica del Instituto Geográfico Nacional (10). Relata los sismos de intensidad igual o superior a VIII, según la Escala Macrosísmica Europea 1998 EMS-98 ${ }^{1}$ (11), ocurridos entre los siglos XIV a XIX, ambos inclusive, considerándose que la información relativa a este período de seiscientos años se puede analizar con ciertas garantías (6).

La Tabla 1 pone de manifiesto tres hechos relevantes:

1) Obviando el llamado terremoto de Lisboa de 1755 y el correspondiente tsunami, la península Ibérica presenta un nivel máximo de la magnitud momento $\mathrm{M}_{\mathrm{w}}=7,0$ (8).

2) Han llegado a transcurrir más de doscientos años entre eventos sísmicos de intensidad igual o superior a VIII en una misma área geográfica (7).

3) No ha habido un sismo de intensidad igual o superior a VIII desde 1884 (terremoto de Arenas del Rey), cuando entre los siglos XIV a XIX el número fue de dos a cinco por siglo. Este hecho ha influido poderosamente, sin duda, en la escasa conciencia sísmica existente en todos los ámbitos de la sociedad española actual, incluidos el docente, en general, y el universitario, en particular.

La Tabla 2 recoge los seísmos más significativos del siglo XX y de lo que va del siglo XXI. El de Dúrcal de 1954, a pesar de tener una magnitud de 7,0, no causó daños por su gran profundidad focal, que fue de $650 \mathrm{~km}$; sin embrago, el de Albolote, con una magnitud de 5,0 , provocó daños importantes por la vulnerabilidad elevada de los edificios afectados. El terremoto de 1969 tuvo unos efectos escasos, a pesar de su magnitud, por la ubicación de su epicentro, si bien hubo 19 muertos: 4 en España, 2 en Portugal y 13 en Marruecos.

El último sismo de la Tabla 2 es el de Lorca de 2011, con una magnitud de 5,1 y una intensidad macrosísmica de VII (12), lo que concuerda con la peligrosidad sísmica prevista normativamente (13). Es cierto, por ejemplo, que la aceleración máxima del terreno fue más de tres veces mayor que la aceleración básica establecida por la norma NCSE-02 (15), que los desplazamientos máximos del terreno se situaron entre los $3 \mathrm{y}$ los $4 \mathrm{~cm}$ y que la rotura de la falla de Alhama de Murcia fue bastante superficial. Sin embrago, la magnitud de los daños registrados y, por supuesto, las nueve víctimas mortales que se produjeron, obliga a profundizar en el análisis de lo ocurrido y, en especial, en las cuestiones relacionadas con la microzonificación sísmica y, sobre todo, con la vulnerabilidad de las construcciones.

\section{ALGUNOS ASPECTOS BÁSICOS DE LA NORMATIVA SISMORRESISTE}

Las normas sísmicas han evolucionado hacia planteamientos probabilistas, pues alcanzar los objetivos del proyecto sólo es parcialmente posible y cuantificable en términos probabilis-

\footnotetext{
${ }^{1}$ La Escala EMS-98 contempla intensidades comprendidas entre I y XII y considera que un terremoto de intensidad igual a VIII es «fuertemente dañino».
} 
Tabla 1.

\begin{tabular}{|c|c|c|c|c|c|}
\hline Siglo & Fecha & Intensidad EMS-98 & Magnitud $M_{w}$ & Localización & Muertos \\
\hline \multirow{3}{*}{ XIV } & $24-08-1356$ & VIII & - & SW. Cabo San Vicente & \\
\hline & 02-03-1373 & VIII-IX & - & Condado de Ribagorça (Huesca-Lleida) & \\
\hline & $18-12-1396$ & VIII-IX & $(6,5)$ & Tavernes de la Valldigna (Valencia) & \\
\hline \multirow{4}{*}{$\mathrm{XV}$} & $15-05-1427$ & VIII-IX & - & Olot (Girona) & \\
\hline & 02-02-1428 & IX-X & - & Queralbs (Girona) & 800 \\
\hline & 24-04-1431 & VIII-IX & $(6,7)$ & Sur de Granada & \\
\hline & 26-01-1494 & VIII & - & Sur de Málaga & \\
\hline \multirow{4}{*}{ XVI } & 05-04-1504 & VIII-IX & $(6,8)$ & Carmona (Sevilla) & 32 \\
\hline & 09-11-1518 & VIII-IX & & Vera (Almería) & 165 \\
\hline & 22-09-1522 & VIII-IX & $(6,5)$ & Mar de Alborán & 1000 \\
\hline & $30-09-1531$ & VIII-IX & & Baza (Granada) & 400 \\
\hline \multirow{3}{*}{ XVII } & 19-06-1644 & VIII & & Muro de Alcoy (Alicante) & 22 \\
\hline & $31-12-1658$ & VIII & & Almería & \\
\hline & $09-10-1680$ & VIII-IX & $(6,8)$ & Alhaurín el Grande (Málaga) & 70 \\
\hline \multirow{2}{*}{ XVIII } & $23-3-1748$ & IX & $(6,2)$ & Estubeny (Valencia) & 38 \\
\hline & 01-11-1755 & $\mathrm{X}$ & $(8,5)$ & SW. Cabo San Vicente & 15.000 \\
\hline \multirow{4}{*}{ XIX } & $25-08-1804$ & VIII-IX & $(6,4)$ & Dalias (Almería) & 407 \\
\hline & 27-10-1806 & VIII & $(5,3)$ & Pinos Puente (Granada) & 13 \\
\hline & 21-03-1829 & IX-X & $(6,6)$ & Torrevieja (Alicante) & 389 \\
\hline & $25-12-1884$ & IX-X & $(6,5)$ & Arenas del Rey (Granada) & 839 \\
\hline
\end{tabular}

(): Magnitud estimada

Tabla 2.

\begin{tabular}{|c|l|c|c|l|c|}
\hline Siglo & Fecha & Intensidad EMS-98 & Magnitud $\mathbf{M}_{\mathbf{w}}$ & Localización & Muertos \\
\hline \multirow{3}{*}{ XX } & $29-03-1954$ & V & 7,0 & Dúrcal (Granada) & \\
\cline { 2 - 6 } & $19-04-1956$ & VII-VIII & 5,0 & Albolote (Granada) & 11 \\
\cline { 2 - 6 } & $28-02-1969$ & VII & 7,8 & SW. Cabo San Vicente & 19 \\
\hline \multirow{2}{*}{ XXI } & $11-05-2011$ & VII & 5,1 & Lorca (Murcia) & 9 \\
\hline
\end{tabular}

tas. La razón es la naturaleza aleatoria de los movimientos sísmicos y el hecho de que los recursos de que se dispone siempre son limitados (14).

Los objetivos clásicos del diseño sismorresistente son la protección de las vidas humanas, la limitación de los daños económicos y el mantenimiento de la operatividad de las construcciones, equipos e instalaciones que sean importantes para la protección civil (15) (16). Tales objetivos se traducen en dos requisitos fundamentales, que son el de no colapso y el de limitación de los daños.

El objetivo del requisito fundamental de no colapso consiste en evitar que se arruine el conjunto de la estructura, o alguna de sus partes, durante un movimiento sísmico poco frecuente; es decir, se trata de lograr que la estructura mantenga su integridad y la suficiente resistencia residual para permitir la evacuación del edificio. No obstante, el coste de la intervención necesaria para que el edificio vuelva a cumplir sus funciones y posea el margen de seguridad requerido puede ser inviable por razones económicas (16) (14).

El requisito fundamental de limitación de los daños está destinado a conseguir que, bajo sismos bastante más frecuentes que los correspondientes al requisito anterior, no haya daños significativos en los elementos no estructurales, o bien limitaciones de uso de los edificios cuyo coste sea desproporcionado (15) (16), siendo uno de los objetivos explícitos de la norma NCSE-02 (15). Para ello, es básico que:

- El sismo no genere deformaciones permanentes significativas en la estructura.

- Todos los elementos estructurales mantengan plenamente su capacidad de respuesta sin intervención alguna.

- Los daños sufridos por los elementos no estructurales permitan una intervención viable desde el punto de vista económico.

Además, se han de adoptar medidas específicas para limitar las incertidumbres y propiciar una respuesta adecuada de la construcción bajo acciones más severas que la acción sísmica de cálculo. Las medidas específicas prescritas por la normativa están destinadas a prevenir un colapso generalizado de la estructura bajo sismos muy poco frecuentes (períodos medios de retorno de 1500 a 2000 años) sin necesidad de incrementar la acción sísmica de cálculo. Sucintamente, las medidas específicas consideradas en la norma EN 1998-1 (16) son las siguientes: 
- Logro de la mayor regularidad posible en horizontal y en vertical.

- Prevención de roturas frágiles y de aparición prematura de mecanismos de colapso de modo que haya una jerarquización adecuada de la resistencia de los elementos estructurales y de las causas de agotamiento.

- Atención especial al proyecto y a la ejecución de las regiones críticas de los elementos estructurales, es decir, de las zonas de las piezas en las que es previsible una respuesta no lineal, pues éstas condicionan en gran medida el comportamiento sísmico de la estructura.

- Uso de modelos de análisis adecuados tomando en consideración, si resulta pertinente, la influencia de la capacidad de deformación del suelo y de los elementos no estructurales.

- Diseño de la cimentación con la rigidez suficiente para lograr que transmita los esfuerzos al terreno con la mayor uniformidad posible.

- Detalle suficiente de los planos del proyecto.

- Definición de las cláusulas de control necesarias y de los métodos de comprobación.

- Redacción de un plan de control específico que abarque el proyecto, la ejecución y el uso si la estructura tiene una importancia especial y se halla ubicada en una zona cuya peligrosidad sísmica sea elevada.

El análisis probabilista de la amenaza sísmica permite generar una curva de peligrosidad que indique cómo varía cierta medida «IM» de la intensidad sísmica, o sea, el parámetro elegido para representar el movimiento sísmico, con respecto a la frecuencia media anual de su superación en un lugar concreto y suponiendo, en general, que el suelo es roca (17).

Los dos requisitos fundamentales se comprueban con magnitudes diferentes de la acción sísmica. Es habitual el uso de la distribución de Poisson para expresar la probabilidad «P» de superación de una magnitud especificada del parámetro «IM» durante el período de tiempo de exposición « $\mathrm{T}_{\mathrm{L}}$ " (años), que se puede tomar igual a la vida útil de la construcción (17) [2].

$$
P=1-e^{-\lambda(I M) T_{L}}
$$

siendo « $\lambda(\mathrm{IM}) »$ la probabilidad anual de superación del valor de referencia de «IM». Y en muchos casos de interés práctico, el producto $\lambda(\mathrm{IM}) \mathrm{T}_{\mathrm{L}}<<1$, de ahí que resulte aceptable la aproximación siguiente (18) [3].

$$
P \approx \lambda(I M) T_{L}
$$

En el ámbito de la normativa técnica, la probabilidad «P»se suele vincular al valor inverso de « $\lambda(\mathrm{IM}) »$, llamado período medio de retorno « $\mathrm{T}_{\mathrm{R}}$ " (años), cuya expresión es la siguiente [4].

$$
T_{R}=\frac{1}{\lambda(I M)}=\frac{-T_{L}}{\ln (1-P)} \approx \frac{T_{L}}{P}
$$

El período medio de retorno representa el intervalo de tiempo en el que cabe esperar que se supere el valor de referencia de «IM» con una probabilidad del 63,2 \% (19) aproximadamente, como se demuestra operando con $T_{L}=T_{R}=1 / \lambda(I M)$ en la ecuación [2].

Si se trata de estructuras ordinarias, la norma EN 1998-1 (16) recomienda que se defina la acción sísmica en función de los criterios siguientes:
- Requisito fundamental de no colapso: $\mathrm{P}=10 \% \mathrm{si} \mathrm{T}_{\mathrm{L}}=50$ años.

- Requisito fundamental de limitación de los daños: $\mathrm{P}=10$ $\%$ si $\mathrm{T}_{\mathrm{L}}=10$ años o, lo que es lo mismo, $\mathrm{P}=40,9 \% \mathrm{si}_{\mathrm{L}}=$ 50 años.

Como se deduce de aplicar la ecuación [4], estos criterios equivalen a establecer la acción sísmica para períodos medios de retorno « $\mathrm{T}_{\mathrm{R}}$ " de 475 años o de 95 años, respectivamente, según se trate de satisfacer el requisito fundamental de no colapso o el requisito fundamental de limitación de los daños.

\section{EL FACTOR DE RIESGO DE LAS CONSTRUCCIONES}

Está ampliamente aceptado en la actualidad que los valores máximos (peak values) de los parámetros que caracterizan el movimiento sísmico no representan bien la severidad de un sismo y sus posibles consecuencias constructivas (14). No obstante, por simplicidad, la norma EN 1998-1 (16) describe y cuantifica la peligrosidad sísmica en función de un valor característico de la aceleración horizontal « $\mathrm{a}_{\mathrm{gR}}$ " de un terreno rocoso o similar (reference peak ground acceleration on type A ground).

La norma española NCSE-O2 (15) basa también la peligrosidad sísmica en un valor característico de la aceleración horizontal de la superficie del terreno denominado aceleración sísmica básica « $\mathrm{a}_{\mathrm{b}}$ ", ponderada con un coeficiente de contribución «K», que sirve para tomar en cuenta la influencia de terremotos derivados de la zona Azores-Gibraltar.

Según NCSE-O2 (15) y EN 1998-1 (16), la aceleración de referencia (básica) se ha de multiplicar por otros dos factores, «S» $\mathrm{y}$ « $\rho »$. El primero es el coeficiente de amplificación del terreno. El segundo es el llamado factor de riesgo " $\rho$ » en NCSE-O2 (15) y factor de importancia " $\gamma_{I}$ " en la norma EN 1998-1 (16) y sirve para modificar la fiabilidad vinculada a la satisfacción de los requisitos fundamentales de no colapso y de limitación de los daños; es decir, se utiliza para variar el período medio de retorno de la acción sísmica en función del uso de la construcción y de las consecuencias de su deterioro o agotamiento. La Tabla 3 recoge y compara la clasificación de las construcciones efectuada por las normas UNE-EN 1990 (20), NCSR-O2 (15) y EN 1998-1 (16).

\subsection{El factor de importancia y el período medio de retorno}

Según EN 1998-1 (16), se puede considerar en muchas ocasiones que la probabilidad anual de superación de la aceleración de referencia del terreno $« \lambda\left(a_{\mathrm{gR}}\right) »$ responde a la siguiente ley potencial: [5].

$$
\lambda\left(a_{g R}\right)=\frac{1}{T_{R}} \approx k_{\mathrm{o}} a_{g R}^{-k}
$$

en la que « $k_{o}>O »$ es una constante que depende de la sismicidad del lugar considerado $\mathrm{y}$ «k $>0$ » es igual a la pendiente de la curva de peligrosidad basada en la aceleración del suelo (16) o en la aceleración espectral (21) (22). Por lo tanto, el coeficiente «k» aporta información acerca de la frecuencia de terremotos más raros o de terremotos más frecuentes en el entorno del parámetro elegido para representar la intensidad sísmica (17).

Se ha recomendado tomar $« \mathrm{k}=3 »(16)$. Este valor puede ser apropiado en zonas de Europa de sismicidad elevada como 
Tabla 3.

\begin{tabular}{|c|c|c|c|}
\hline Norma & UNE-EN 1990 (2003) & NCSE-02 (2002) & EN 1998-1 (2004) \\
\hline \multirow{4}{*}{$\begin{array}{l}\text { Clase de consecuencias, } \\
\text { de riesgo o de importancia }\end{array}$} & Consecuencias leves & Importancia moderada & $\begin{array}{l}\text { I. Poca importancia para la seguridad } \\
\text { pública: } 0,8^{* *}\end{array}$ \\
\hline & Consecuencias moderadas & Importancia normal: 1,0 * & II. Importancia ordinaria: $1, o^{* *}$ \\
\hline & \multirow{2}{*}{ Consecuencias graves } & \multirow{2}{*}{ Importancia especial: 1,3 * } & $\begin{array}{l}\text { III. Importancia elevada: } 1,2 * \text { (escuelas, salones } \\
\text { de actos, instituciones culturales, etc.) }\end{array}$ \\
\hline & & & $\begin{array}{l}\text { IV. Importancia vital: } 1,4 * \text { (hospitales, parques } \\
\text { de bomberos, cen-trales energéticas, etc.) }\end{array}$ \\
\hline
\end{tabular}

* factor de riesgo ${ }^{* *}$ factor de importancia

Italia, aunque se trata de una simple aproximación a la realidad (14):

- Corresponden valores menores de «k» a las zonas en las que la sismicidad la determinan sucesos de intensidad importante muy espaciados en el tiempo.

- Son adecuados valores mayores de «k» en las zonas en las que los terremotos son frecuentes, o cuando se consideran períodos de tiempo de reducidos a intermedios o valores elevados de la aceleración espectral.

Aplicando la ecuación [5] a construcciones de clase distinta a la II (Tabla 3), se tiene [6].

$$
\lambda\left(\gamma_{I} a_{g R}\right)=\frac{1}{T_{R}\left(\gamma_{I}\right)} \approx k_{\mathrm{o}}\left(\gamma_{I} a_{g R}\right)^{-k}
$$

donde « $T_{R}\left(\gamma_{I}\right)$ » representa el período medio de retorno correspondiente a una aceleración igual a $" \gamma_{\mathrm{I}} \mathrm{a}_{\mathrm{gR}}$ ". Dividiendo ahora miembro a miembro las ecuaciones [5] y [6], resulta:

de donde

$$
\frac{1 / T_{R}}{1 / T_{R}\left(\gamma_{I}\right)} \approx \frac{k_{\mathrm{o}} a_{g R}^{-k}}{k_{\mathrm{o}}\left(\gamma_{I} a_{g R}\right)^{-k}}
$$

$$
T_{R}\left(\gamma_{I}\right) \approx \gamma_{I}^{k} T_{R}
$$

expresión que permite estimar el período medio de retorno para cada valor del factor de importancia $« \gamma_{I} »(« \rho »$ en NCSE02) (Tabla 4) (Figura 1).

De la ecuación [7] resulta inmediato deducir la ecuación [8], que sirve para calcular $" \gamma_{\mathrm{I}}$ " en función del período medio de retorno « $\mathrm{T}_{\mathrm{R}}$ " de las construcciones ordinarias y del período medio de retorno « $\mathrm{T}_{\mathrm{R}}\left(\gamma_{\mathrm{I}}\right) »$ que se asigne, en un caso concreto, a una obra no ordinaria:

$$
\gamma_{I} \approx\left[\frac{T_{R}}{T_{R}\left(\gamma_{I}\right)}\right]^{-1 / k}
$$

\subsection{El factor de importancia y el período de exposición}

Si se mantiene constante la probabilidad «P» de superación del parámetro de intensidad «IM», el período de exposición « $\mathrm{T}_{\mathrm{L}}$ » que corresponde a las edificaciones ordinarias tiene que adoptar otro valor « $T_{L}\left(\gamma_{I}\right) »$ para las clases de construcción restantes. De acuerdo con las ecuaciones [4] y [7]:

$$
\left.\begin{array}{c}
T_{R}=\frac{-T_{L}}{\ln (1-P)} \\
\left(\gamma_{I}\right)=\frac{-T_{L}\left(\gamma_{I}\right)}{\ln (1-P)}
\end{array}\right\} \frac{T_{R}\left(\gamma_{I}\right)}{T_{R}}=\frac{T_{L}\left(\gamma_{I}\right)}{T_{L}} \rightarrow \frac{\gamma_{I}^{k} T_{R}}{T_{R}}=\frac{T_{L}\left(\gamma_{I}\right)}{T_{L}}
$$

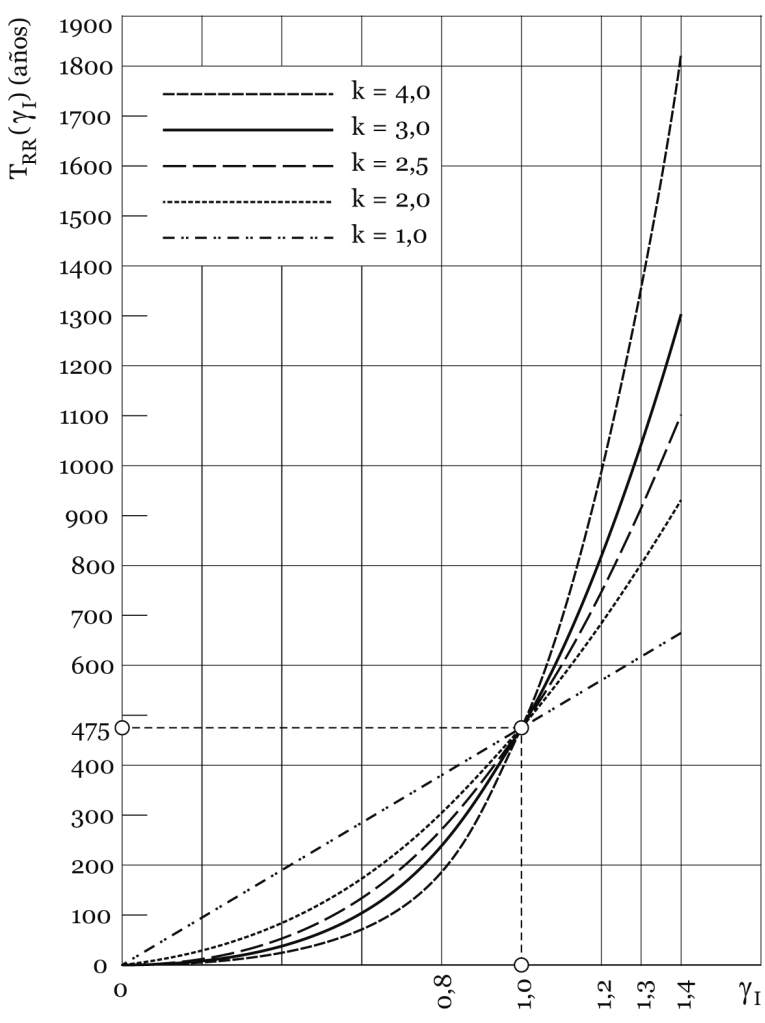

Figura 1. Período de retorno y factor de importancia. Requisito fundamental de no colapso.

de donde:

$$
T_{L}\left(\gamma_{I}\right)=\gamma_{I}^{k} T_{L}
$$

$\mathrm{y}$

$$
\gamma_{I}=\left[\frac{T_{L}}{T_{L}\left(\gamma_{I}\right)}\right]^{-1 / k}
$$

que es la primera propuesta contenida en la Nota al punto (4) del apartado 2.1 de EN 1998-1 (16).

\subsection{El factor de importancia y la probabilidad de superación}

Si se mantiene constante el período de exposición « $\mathrm{T}_{\mathrm{L}}$ ", la probabilidad «P» de superación del parámetro de intensidad «IM» que corresponde a las edificaciones ordinarias tiene que adoptar un valor distinto «P $\left(\gamma_{\mathrm{I}}\right) »$ para las restantes clases de construcción (Tabla 3). De acuerdo con las ecuaciones [4] y [7]:

$$
\left.\begin{array}{c}
T_{R}=\frac{-T_{L}}{\ln (1-P)} \\
T_{R}\left(\gamma_{I}\right)=\frac{-T_{L}}{\ln \left[\left(1-P\left(\gamma_{I}\right)\right]\right.}
\end{array}\right\} \frac{T_{R}\left(\gamma_{I}\right)}{T_{R}}=\frac{\ln (1-P)}{\ln \left[\left(1-P\left(\gamma_{I}\right)\right]\right.} \rightarrow
$$


Tabla 4 .

\begin{tabular}{|c|c|c|c|c|c|}
\hline \multirow{2}{*}{ Clasificación de la construcción } & \multicolumn{5}{|c|}{ Período medio de retorno (años) } \\
\hline & $\mathbf{k}=\mathbf{1}, \mathbf{o}^{\mathbf{c})}$ & $\mathbf{k}=\mathbf{2 ,} \mathbf{o}^{\mathbf{c})}$ & $\mathbf{k}=\mathbf{2}, \mathbf{5}^{\mathrm{d})}$ & $\mathbf{k}=\mathbf{3}, \mathbf{o}^{\mathbf{c}) \mathbf{d})}$ & $k=4, o^{c) d)}$ \\
\hline I: $\quad \gamma_{I}=0,8^{a}$ & 380 & 304 & 272 & 243 & 195 \\
\hline II: $\gamma_{I}=1,0^{a) b)}$ & 475 & 475 & 475 & 475 & 475 \\
\hline III: $\gamma_{I}=1,2^{a)}$ & 570 & 684 & 749 & 821 & 985 \\
\hline$\gamma_{I}=1,3^{\mathrm{b})}$ & 618 & 803 & 915 & 1044 & 1.357 \\
\hline IV: $\gamma_{I}=1,4^{a)}$ & 665 & 931 & 1.102 & 1.303 & 1.825 \\
\hline
\end{tabular}

${ }^{a)}$ EN 1998-1; ${ }^{b)}$ NCSE-O2; ${ }^{c}$ FEMA-35O y FEMA-351; ${ }^{d)} 14$

$$
\rightarrow \frac{\gamma_{I}^{k} T_{R}}{T_{R}}=\frac{\ln (1-P)}{\ln \left[\left(1-P\left(\gamma_{I}\right)\right]\right.} \approx \frac{P}{P\left(\gamma_{I}\right)}
$$

de donde:

$$
P\left(\gamma_{I}\right) \approx \frac{P}{\gamma_{I}^{k}}
$$

$\mathrm{y}$

$$
\gamma_{I} \approx\left[\frac{P\left(\gamma_{I}\right)}{P}\right]^{-1 / k}
$$

que es la segunda propuesta contenida en la Nota al punto (4) del apartado 2.1 de EN 1998-1 (16).

\subsection{El factor de importancia, el factor de reducción y la limitación de daños}

La comprobación del requisito fundamental de limitación de los daños también se ha de efectuar teniendo en cuenta la clase de construcción mediante el correspondiente factor de importancia. Además, la opinión más extendida internacionalmente es que la manera más eficaz y segura de controlar la magnitud de los daños consiste en limitar los desplazamientos y distorsiones de los elementos estructurales y no estructurales (23). Para la consecución de tal objetivo se debe atender a las cuestiones siguientes:

a) No hay que obviar las características de los elementos no estructurales, como, por ejemplo, su resistencia, su rigidez y su fragilidad.

b) El sistema global es tanto menos sensible a la interacción estructura-elementos no estructurales cuanto más rígida es la estructura. c) La limitación adecuada de los daños suele controlar la escuadría de las barras de los pórticos de hormigón, de acero o mixtos si no hay otros elementos estructurales de rigidez mayor (24) (14).

d) En España es muy frecuente que los edificios tengan estructuras reticulares, con frecuencia de hormigón armado, y cerramientos y particiones constituidas por fábricas no armadas. Esta solución constructiva, en el caso de las fachadas sobre todo, ha sido cuestionada seria y muy fundadamente cuando las acciones son de naturaleza gravitatoria (25) (26) o, con mucho mayor motivo, cuando son de origen sísmico (27) (28).

e) Los sistemas mencionados en el punto anterior y, en general, los constituidos por soluciones estructurales de rigidez limitada y fábricas no armadas, suelen ser muy sensibles a aportes relativamente reducidos de energía sísmica, como los correspondientes a la limitación de los daños (29); tanto más cuanto mayor es la duración del movimiento sísmico, cuestión no suficientemente valorada en muchos casos. Así lo han demostrado de manera reiterada seísmos como los de Atenas (1999), Kocaeli (1999), Gujarat (2001), Bingol (2003), Boumerdès (2003), L’Aquila (2009), etc., y, por supuesto, el de Lorca de 2011 (Figura 2).

En coherencia con estas consideraciones, EN 1998-1 (16) limita el desplome local relativo « $\mathrm{d}_{\mathrm{ri}} / \mathrm{h}_{\mathrm{i}}$ » de cada planta del modo siguiente:

$$
\frac{d_{r i}}{h_{i}} \leq \frac{\xi}{v}
$$

siendo:
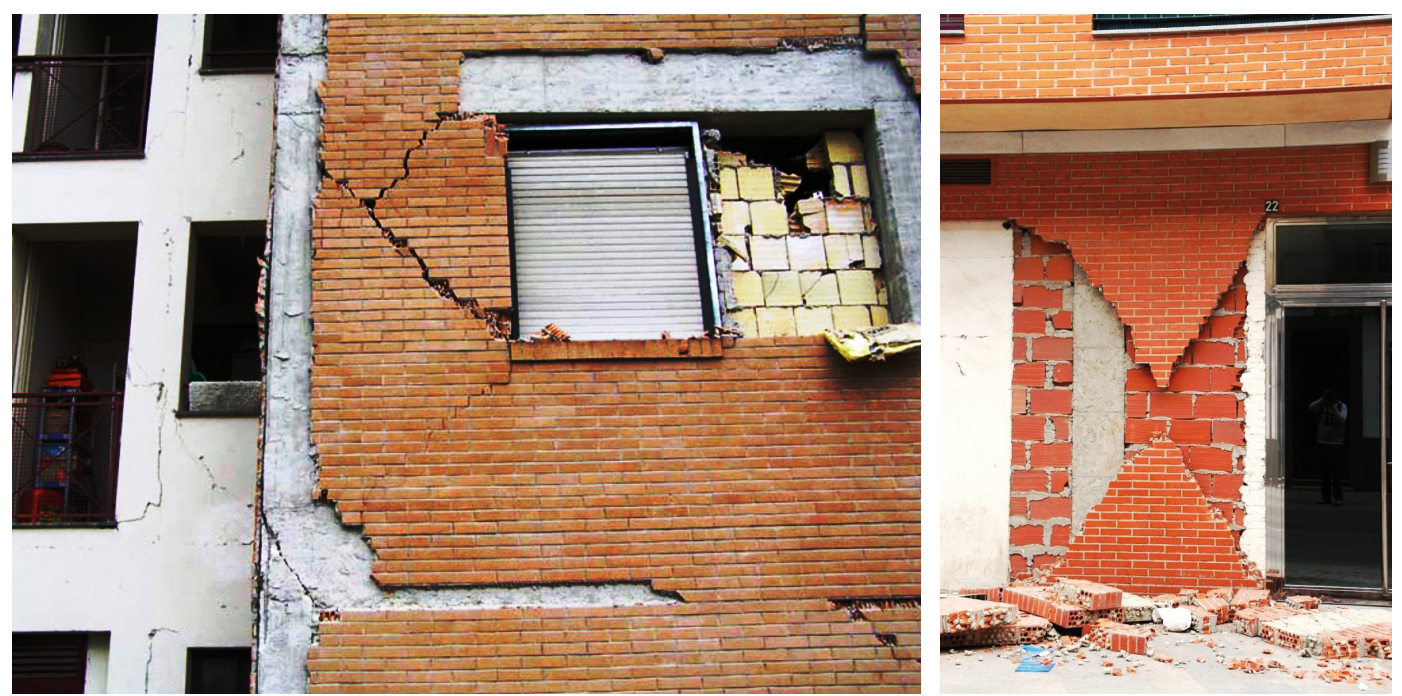

Figura 2. Daños en fachadas: izq.: L’Aquila, 2009; drch: Lorca, 2011. 
$\mathrm{d}_{\mathrm{ri}}$ diferencia entre los desplazamientos laterales de los forjados que delimitan la planta «i» calculados con la acción sísmica del requisito fundamental de no colapso

$\mathrm{h}_{\mathrm{i}} \quad$ altura de la planta «i»

$\xi$ parámetro que sirve para tomar en consideración la fragilidad de los elementos no estructurales y su potencial interacción con la estructura, que adopta los valores siguientes:

- 0,0050: elementos no estructurales frágiles y vinculados a la estructura

- 0,0075: elementos no estructurales dúctiles

- 0,0100: elementos no estructurales dúctiles y no vinculados a la estructura

$v$ factor de reducción para tener en cuenta el menor período de retorno asociado a la satisfacción del requisito de limitación de los daños, recomendando asignarle los valores siguientes a falta de una información más precisa:

- 0,50 clases de construcción I y II (Tabla 3)

- o,40 clases de construcción III y IV (Tabla 3)

\section{CONCLUSIONES}

1. La peligrosidad sísmica de la península Ibérica no es despreciable, con un nivel máximo de la magnitud momento $\mathrm{M}_{\mathrm{w}}=7,0$. Los terremotos más destructivos ocurrieron antes del siglo XX, por lo que la conciencia sísmica de la sociedad española y de sus instituciones es, en general, insuficiente.

2. Las normas sísmicas actuales son probabilistas, dado que los seísmos y las respuestas de las construcciones son fenómenos aleatorios y que los recursos siempre son limitados.
3. La norma EN 1998-1 recomienda períodos medios de retorno de la acción sísmica de 475 años para el requisito fundamental de no colapso y de 95 años para el requisito fundamental de limitación de los daños si se trata de estructuras ordinarias.

4. Las medidas específicas de las normas equivalen, de hecho, a establecer un nuevo requisito fundamental para terremotos muy poco probables; es decir, para períodos medios de retorno mucho más amplios.

5. El factor de importancia (EN 1998-1) (de riesgo en NCSEo2) permite modificar la fiabilidad de la comprobación de los requisitos fundamentales en función de la importancia y uso de cada construcción.

6. Partiendo de los modelos que representan las ecuaciones [2] y [5], se han deducido las ecuaciones [8], [10] y [12]. Éstas relacionan el factor de importancia con el período medio de retorno, con el período de exposición y con la probabilidad de superación del parámetro de referencia de la intensidad sísmica, respectivamente.

7. El enorme impacto humano y económico de terremotos recientes ha demostrado que hay que poner el énfasis en la microzonificación sísmica y en la vulnerabilidad de las construcciones existentes y futuras; sobre todo, por lo que a la limitación de los daños respecta, lo que hace necesario establecer un control específico de las características y de la rigidez de los sistemas.

8. Como escribió Jhon Filson (30), científico emérito del Servicio Geológico de los Estados Unidos:

«Natural hazards are inevitable. Natural disasters are not»

\section{REFERENCIAS}

(1) Blázquez, R. (2011). Ciencia y conciencia sísmica en España. Zuncho, (30): 5-11, Especial sismo.

(2) Laban-Mattei, O. (2012, 14 de junio). Desastres naturales: más de un millón de muertos en los últimos 20 años. RT (Russia Today).

(3) Muñoz, D. (1989). Conceptos básicos en riesgo sísmico. Física de la Tierra, 1: 199-215.

(4) UNDRO (Office of the United Nations Disaster Relief Co-ordinator). (1980). Natural disasters and vulnerability analysis, Report of Expert Group Meeting. Ginebra. http://archive.org/stream/naturaldisastersoooffi/naturaldisastersoooffi_djvu.txt.

(5) Blázquez-Martínez, R. (1997). Enfoque y avances conceptuales de la nueva norma española de construcción sismorresistente NCSE-94. Informes de la Construcción, 48(447): 39-45, doi: http://dx.doi.org/10.3989/ic.1997.v48.i447.974.

(6) Martínez-Solares, J.M. (2001). Los efectos en España del terremoto de Lisboa (1 de noviembre de 1755). Monografía núm. 19. Madrid: Ministerio de Fomento - Dirección General del Instituto Geográfico Nacional. http://www.ign.es/ign/ resources/sismologia/publicaciones/EfectosEspanaterremotoLisboa.pdf.

(7) Martínez-Solares, J.M. (2003). Sismicidad histórica de la Península Ibérica. Física de la tierra, 15: 13-28. http://revistas.ucm.es/index.php/FITE/article/view/FITE0303110013A.

(8) Martínez-Solares, J.M. (2012). Sismicidad pre-instrumental. Los grandes terremotos históricos en España. Enseñanza de las Ciencias de la Tierra, 19(3): 296-304.

(9) Martínez-Solares, J.M., Mezcua, J. (2001). Catálogo sísmico de la Península Ibérica (880 a.C.-19oo). Monografía núm. 18. Madrid: Ministerio de Fomento - Dirección General del Instituto Geográfico Nacional. http://www.ign.es/ign/resources/sismologia/publicaciones/Catalogohasta190o.pdf

(10) IGN. (2012). Listado de los terremotos más importantes en España. Madrid: Instituto Geográfico Nacional - Servicio de Información Sísmica. http://www.ign.es/ign/layoutIn/sismoTerremotosEspana.do?value=1.

(11) G. Grünthal (Ed.) (2008). Escala Macrosísmica Europea ESC-98. Hamburgo: Comisión Sismológica Europea. (Edición española: F. Lorenzo Martín)

(12) Cabañas, L., Carreño, E., Izquierdo, A., Martínez-Solares, J.M., Capote-del Villar, R., Martínez-Díaz, J., Benito, B., Gaspar, J., Rivas, A., García-Mayordomo, J., Pérez-López, R., Rodríguez-Pascua, M. A., Murphy, P. (2011). Informe del sismo de Lorca del 11 de mayo de 2011. Madrid: Instituto Geográfico Nacional. http://hdl.handle.net/10261/62381.

(13) Barbat, A.H. (2011). Lorca, el terremoto improbable. Zuncho, (30): 13-19, Especial sismo.

(14) Carvalho, E.C. (2011). Overview of Eurocode 8. Lisbon: Eurocode 8, Background \& Applications. http://eurocodes.jrc. ec.europa.eu/doc/WS_335/S1_EC8-Lisbon_E\%20CARVALHO.pdf. 
(15) NCSE-02. (2002). Real Decreto 997/2002, de 27 de septiembre, por el que se aprueba la Norma de Construcción Sismorresistente: Parte General y de Edificación (NCSE-02). Boletín Oficial del Estado, (244): 35898-35967. España: Ministerio de Fomento.

(16) CEN. (2004). EN 1998-1:2004 Eurocode 8. Design of structures for earthquake resistance. General rules, seismic actions and rules for buildings. Comité Europeo de Normalización.

(17) FIB. (2012). Probabilistic performance-based seismic design. Fib bulletin, $\mathrm{n}^{\mathrm{0}} 68$ (Technical Report). Lausanne.

(18) CSN. (2008). DID-16.o8 Proyecto Expel: Sistema experto de análisis probabilista de la peligrosidad sísmica. Madrid: Consejo de Seguridad Nuclear. http://www.csn.es/images/stories/publicaciones/unitarias/documentos_id/expel.pdf.

(19) Benito. B, Jiménez, E. (1999). Peligrosidad sísmica. Física de la tierra, 11: 13-47.

(20) AENOR. (2003). UNE-EN 199o. Eurocódigos. Bases de cálculo de estructuras. Asociación Española de Normalización.

(21) SAC Joint Venture. (2000). Recommended Seismic Design Criteria for New Steel Moment-Frame Buildings. Washington D.C.: SAC Joint Venture for the Federal Emergency Management Agency. http://www.nehrp.gov/pdf/fema350.pdf.

(22) SAC Joint Venture. (2000). Recommended Seismic Evaluation and Upgrade Criteria for Existing Welded Steel Moment-Frame Buildings. Washington D.C.: SAC Joint Venture for the Federal Emergency Management Agency. http:// www.nehrp.gov/pdf/fema351.pdf.

(23) Perepérez, B. (2011). La configuración sísmica de los sistemas. Zuncho, (30): 21-28, Especial sismo.

(24) Fardis, M.N. (2004, 28 de junio - 1 de julio). A european perspective to performance-based seismic design, assessment and retrofitting. Performance-based seismic design concepts and implementation, 1-13. En Proceedings of the International Workshop in Bled, Slovenia. Berkeley: Pacific Earthquake Engineering Research Center - College of Engineering, University of California.

(25) Adell, J.M., Vega, S. (2005). La fachada contemporánea con ladrillo: cerramientos tipo. Informes de la Construcción, 56(495): 13-31, doi: http://dx.doi.org/10.3989/ic.2005.v57.i495.452.

(26) Ros-García, J.M. (2005). La fábrica de doble hoja en Madrid, un siglo de cerramiento moderno. Informes de la Construcción, 56(495): 57-71, doi: http://dx.doi.org/ 10.3989/ic.2005.v57.i495.455.

(27) Álvarez-Cabal, R., Dávila, S., Díaz-Pavón, E. (2011). Comportamiento de los cerramientos y particiones durante el terremoto de Lorca. Zuncho, (30): 29-43, Especial sismo.

(28) Dávila, S., Hoogendoorn, P.P., Álvarez-Cabal, R. (2011). Acciones en fachadas. Cuadernos Intemac, (83): 1-39.

(29) Calvi, G.M., Bolognini, D., Penna, A. (2004, 14-16 de abril). Seismic performance of masonry-infilled R.C. frames: benefits of slight reinforcements. En SÍSMICA 2004 - $6^{\circ}$ Congreso Nacional de Sismologia e Engenharia Sísmica. Guimaraes.

(30) Filson, J.R. (2004, 28 de diciembre). To the Editor. Re «A Day of Devastation» (editorial, Dec. 27). New York Times. 